\title{
Pacific
}

Journal of

Mathematics

ON THE EXTENSION OF LIPSCHITZ FUNCTIONS FROM BOUNDARIES OF SUBVARIETIES TO STRONGLY PSEUDOCONVEX DOMAINS

K. AdACHI AND HiRoshi KaJimoto 


\section{ON THE EXTENSION OF LIPSCHITZ FUNCTIONS FROM BOUNDARIES OF SUBVARIETIES TO STRONGLY PSEUDOCONVEX DOMAINS}

\section{Kenzō Adachi and Hiroshi KaJimoto}

In this paper, we study the principal value integral on boundaries of subvarieties in strongly pseudoconvex domains and using it, we give a condition for the extendability of Lipschitz functions.

Introduction. Let $D$ be a strongly pseudoconvex domain in $C^{n}$ with $C^{\infty}$ boundary. Henkin [6] and Ramírez [12] obtained independently the support function $g(\zeta, z)$ for $D$ which depends holomorphically on $z$, and then, using this support function, they obtained the integral formula for holomorphic functions in $\bar{D}$. On the other hand, Stout [14], when $p=1$, and then, Hatziafratis [5], when $p$ is arbitrary, obtained the integral formula for a certain subvariety $V$ of codimension $p$ in $D$. By using the support function $g(\zeta, z)$ and the integral formula for $V$, we can obtain the kernel $\Omega(\zeta, z)$ for $(\zeta, z) \in \partial V \times \bar{D}$. In this paper, we shall define the principal value integral P.V. $\int_{\partial V} f(\zeta) \Omega(\zeta, z)$ for a Lipschitz function $f$ on $\partial V$ and $z \in \partial V$. The definition of the principal value integral is the same as that of Alt [2] when $V=D$ (cf. Dolbeault [4]). By using the principal value integral we can give the condition for a Lipschitz function on $\partial V$ to be the boundary value of a function that is holomorphic in $D$ and continuous on $\bar{D}$. Finally we end the introduction by giving an example which shows that the Lipschitz continuity is necessary in order to define the principal value integral.

Example. Define $\varphi \in C^{\infty}(0, \infty)$ such that

$$
\varphi(\theta)= \begin{cases}1 & \text { if } 0<\theta \leq \frac{\pi}{4}, \\ 0 & \text { if } \theta \geq \frac{\pi}{2}\end{cases}
$$

Extend $\varphi$ to an odd function on $R \mid\{0\}$. Let $D$ be the unit disc in $\mathbb{C}$ and $f$ be a function on $\partial D$ such that

$$
f\left(e^{i \theta}\right)= \begin{cases}\frac{\varphi(\theta)}{\log |\theta|} & \text { if } 0<|\theta| \leq \pi \\ 0 & \text { if } \theta=0\end{cases}
$$


Then $f$ is continuous but not Lipschitz continuous on $\partial D$. Compute the principal value integral at $1 \in \partial D$. We find

$$
\begin{aligned}
\mathrm{P} . \mathrm{V} . & \frac{1}{2 \pi i} \int_{|\zeta|=1} \frac{f(\zeta) d \zeta}{\zeta-1} \\
& =\lim _{\varepsilon \rightarrow 0+} \frac{1}{2 \pi} \int_{\varepsilon \leq|\theta| \leq \pi} \frac{f\left(e^{i \theta}\right) e^{i \theta}}{e^{i \theta}-1} d \theta \\
& =\lim _{\varepsilon \rightarrow 0+} \frac{1}{2 \pi} \int_{\varepsilon}^{\pi} f\left(e^{i \theta}\right) \frac{e^{i \theta}+1}{e^{i \theta}-1} d \theta \\
& =\lim _{\varepsilon \rightarrow 0+} \frac{1}{2 \pi} \int_{\varepsilon}^{\pi / 4} \frac{1}{\log \theta} \frac{e^{i \theta}+1}{e^{i \theta}-1} d \theta+\text { (finite value). }
\end{aligned}
$$

But we have

$$
\frac{1}{\log \theta} \frac{e^{i \theta}+1}{e^{i \theta}-1} \sim \frac{-2 i}{\theta \log \theta}
$$

when $\theta \rightarrow 0+$. This shows that the principal value integral of $f$ at 1 does not converge.

1. The integral formula on subvarieties. Let $D$ be a bounded strongly pseudoconvex domain in $C^{n}$ with $C^{\infty}$ boundary. Let $\rho$ be a defining function of $D$, i.e., $D=\{z: \rho(z)<0\}$. We set

$$
\begin{aligned}
F(\zeta, z)= & \sum_{i=1}^{n} 2 \frac{\partial \rho}{\partial \zeta_{i}}(\zeta)\left(z_{i}-\zeta_{i}\right) \\
& +\sum_{i, j=1}^{n} \frac{\partial^{2} \rho}{\partial \zeta_{i} \partial \zeta_{j}}(\zeta)\left(z_{i}-\zeta_{i}\right)\left(z_{j}-\zeta_{j}\right)
\end{aligned}
$$

According to the construction of Henkin [6] (cf. Henkin-Leiterer [8], p. 108), there exists a pseudoconvex neighborhood $\widetilde{D}$ of $\bar{D}$, a neighborhood $W$ of $\partial D$, and a $C^{\infty}$ function $g: W \times \widetilde{D} \rightarrow C$ such that for each $\zeta \in W, g(\zeta, z)$ is holomorphic in $\widetilde{D}$. For $r>0$, define $\Delta_{r}=\{(\zeta, z) \in W \times \widetilde{D}:|\zeta-z|<r\}$. Then there exist a constant $\sigma_{1}>0$ and a non-vanishing $C^{\infty}$ function $Q(\zeta, z)$ on $\Delta_{\sigma_{1}}$ such that $g(\zeta, z)=F(\zeta, z) Q(\zeta, z)$ for $(\zeta, z) \in \Delta_{\sigma_{1}}$ and $g(\zeta, z) \neq 0$ for $(\zeta, z) \in W \times \widetilde{D} \mid \Delta_{\sigma_{1}}$. Moreover $g(\zeta, z)$ admits a division

$$
g(\zeta, z)=\sum_{j=1}^{n} g_{j}(\zeta, z)\left(z_{j}-\zeta_{j}\right)
$$

with $g_{j}: W \times \widetilde{D} \rightarrow C$ of class $C^{\infty}$ and holomorphic in the second variable. Let $h_{1}, \ldots, h_{p}(p<n)$ be holomorphic functions in $\widetilde{D}$. 
Define

$$
\widetilde{V}=\left\{z \in \widetilde{D}: h_{1}(z)=\cdots=h_{p}(z)=0\right\}, \quad V=\widetilde{V} \cap D .
$$

Assume that

$$
\partial h_{1} \wedge \cdots \wedge \partial h_{p} \wedge \partial \rho \neq 0 \text { on } \partial V
$$

By Hefer's theorem, there exist holomorphic functions $h_{i j}(\zeta, z)$ for $(\zeta, z) \in \widetilde{D} \times \widetilde{D}$ such that

$$
h_{i}(\zeta)-h_{i}(z)=\sum_{j=1}^{n} h_{i j}(\zeta, z)\left(\zeta_{j}-z_{j}\right), \quad i=1, \ldots, p
$$

Define

$$
\begin{aligned}
& \alpha^{h}(\zeta, z)=\left|\begin{array}{ccccccc}
g_{1} & h_{11} & \ldots & h_{p 1} & \bar{\partial}_{\zeta} g_{1} & \ldots & \bar{\partial}_{\zeta} g_{1} \\
\vdots & \vdots & & \vdots & \vdots & & \vdots \\
g_{n} & h_{1 n} & \ldots & h_{p n} & \bar{\partial}_{\zeta} g_{n} & \ldots & \bar{\partial}_{\zeta} g_{n}
\end{array}\right| \\
& |\nabla h(\zeta)|^{2}=\sum_{1 \leq j_{1}<\cdots<j_{p} \leq n}\left|\frac{\partial\left(h_{1}, \ldots, h_{p}\right)}{\partial\left(\zeta_{j_{1}}, \ldots, \zeta_{j_{p}}\right)}(\zeta)\right|^{2} \\
& \beta^{h}(\zeta)=(-1)^{p(p+1) / 2}|\nabla h(\zeta)|^{-2}\left|\begin{array}{cccccc}
\overline{\frac{\partial h_{1}}{\partial \zeta_{1}}} & \ldots & \frac{\partial h_{p}}{\partial \zeta_{1}} & d \zeta_{1} & \ldots & d \zeta_{1} \\
\vdots & & \vdots & \vdots & & \vdots \\
\frac{\partial h_{1}}{\partial \zeta_{n}} & \ldots & \frac{\partial h_{p}}{\partial \zeta_{n}} & d \zeta_{n} & \ldots & d \zeta_{n}
\end{array}\right|
\end{aligned}
$$

and

$$
K_{V}(\zeta, z)=c(n, p) \alpha^{h}(\zeta, z) \wedge \beta^{h}(\zeta)
$$

where

$$
c(n, p)=(-1)^{p(n+1)}(-1)^{n(n-1) / 2} \frac{1}{(n-p) !(2 \pi i)^{n-p}} .
$$

Let $n-p=k$. We define the kernel $\Omega(\zeta, z)$ by

$$
\Omega(\zeta, z)=\frac{K_{V}(\zeta, z)}{g(\zeta, z)^{k}}
$$

Let $A(D)$ (resp. $A(V)$ ) be the space of functions that are holomorphic in $D$ (resp. $V$ ) and continuous on $\bar{D}$ (resp. $\bar{V}$ ). Then Hatziafratis [5] proved the following.

THEOREM 1. For $f \in A(V)$ and $z \in V$, the integral formula

$$
f(z)=\int_{\partial V} f(\zeta) \Omega(\zeta, z)
$$

holds.

Now we begin by proving the following lemma. 
LEMMA 1. If $h_{1}(z)=z_{k+1}, \ldots, h_{p}(z)=z_{n}$, then we have

$$
\begin{aligned}
K_{V}(\zeta, z)= & (-1)^{k(k-1) / 2} \frac{(k-1) !}{(2 \pi i)^{k}} \\
& \cdot \sum_{j=1}^{k}(-1)^{j-1} g_{j} \bigwedge_{\substack{i=1 \\
i \neq j}}^{k} \bar{\partial}_{\zeta} g_{i} \wedge d \zeta_{1} \wedge \cdots \wedge d \zeta_{k} .
\end{aligned}
$$

Proof. By the definition of $\alpha^{h}, \beta^{h}$, we have

$$
\begin{aligned}
& \alpha^{h}(\zeta, z)=\left|\begin{array}{cccc}
g_{1} & 0 \cdots 0 & \bar{\partial}_{\zeta} g_{1} \cdots \bar{\partial}_{\zeta} g_{1} \\
\vdots & \vdots & \vdots & \\
g_{p} & 0 \cdots 0 & \vdots \\
\vdots & 1 \cdots 0 & \vdots \\
g_{n} & 0 \cdots 1 & \bar{\partial}_{\zeta} g_{n} \cdots \bar{\partial}_{\zeta} g_{n}
\end{array}\right|=(-1)^{p}\left|\begin{array}{cc}
g_{1} & \bar{\partial}_{\zeta} g_{1} \cdots \bar{\partial}_{\zeta} g_{1} \\
\vdots & \vdots \\
g_{k} & \bar{\partial}_{\zeta} g_{k} \cdots \bar{\partial}_{\zeta} g_{k}
\end{array}\right| \\
& =(-1)^{n-k}(k-1) ! \sum_{j=1}^{k}(-1)^{j-1} g_{j} \bigwedge_{i \neq j} \bar{\partial}_{\zeta} g_{i} \text {, }
\end{aligned}
$$

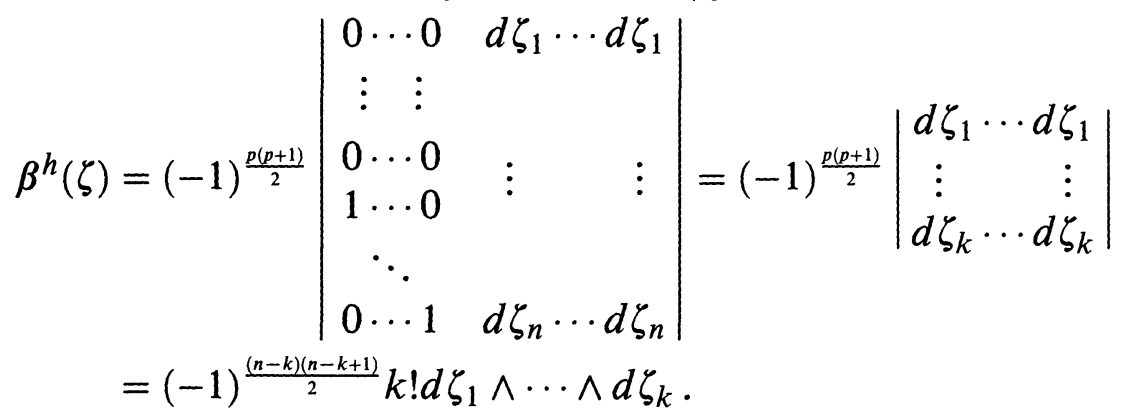

Therefore we have

$$
\begin{aligned}
K_{V}(\zeta, z) & =(-1)^{(n-k)(n+1)}(-1)^{n(n-1) / 2} \frac{1}{k !(2 \pi i)^{k}} \alpha^{h}(\zeta, z) \wedge \beta^{h}(\zeta, z) \\
& =(-1)^{k(k-1) / 2} \frac{(k-1) !}{(2 \pi i)^{k}} \sum_{j=1}^{k}(-1)^{j-1} g_{j} \bigwedge_{i \neq j} \bar{\partial}_{\zeta} g_{i} \wedge d \zeta_{1} \wedge \cdots \wedge d \zeta_{k}
\end{aligned}
$$

This completes the proof of Lemma 1.

For $\sigma>0$, we set $S_{\zeta, \sigma}=\{z:|z-\zeta|<\sigma\}$. Then we have the following. 
LEMMA 2. Let $\sigma, 0<\sigma<\sigma_{1}$, be sufficiently small. Then for $\zeta \in \partial V, z \in \widetilde{D} \cap S_{\zeta, \sigma}$, we can choose a local coordinate system in such a way that

$$
\Omega(\zeta, z)=\frac{2^{k}}{(2 \pi i)^{k}} \frac{\partial \rho(\zeta) \wedge(\bar{\partial} \partial \rho)^{k-1}(\zeta)+e(\zeta, z)}{F(\zeta, z)^{k}},
$$

where $e(\zeta, z)$ is a $(k, k-1)$ form satisfying $e(\zeta, z)=O(|\zeta-z|)$.

Proof. By a local holomorphic change of coordinates, we may assume that $h_{1}=z_{k+1}, \ldots, h_{p}=z_{n}$. We have (cf. Anderson and Berndtsson [3], Lemma 3),

$$
\begin{aligned}
& \sum_{j=1}^{k}(-1)^{j-1} \frac{g_{j} \wedge_{i \neq j} \bar{\partial}_{\zeta} g_{i} \wedge d \zeta_{1} \wedge \cdots \wedge d \zeta_{k}}{g(\zeta, z)^{k}} \\
& =c(k) \frac{\left(\sum_{j=1}^{k} g_{j} d \zeta_{j}\right) \wedge\left(\sum_{j=1}^{\kappa} \bar{\partial}_{\zeta} g_{j} \wedge d \zeta_{j}\right)^{k-1}}{g(\zeta, z) 6 k},
\end{aligned}
$$

where $c(k)=(-1)^{k(k-1) / 2} \frac{1}{(k-1) !}$.

If $|\zeta-z|<\sigma$, then we obtain

$$
\begin{aligned}
& \sum_{i=1}^{n} g_{i}(\zeta, z)\left(z_{i}-\zeta_{i}\right) \\
& \quad=\sum_{i=1}^{n}\left\{\left(2 \frac{\partial \rho}{\partial \zeta_{i}}(\zeta)+\sum_{j=1}^{n} \frac{\partial^{2} \rho}{\partial \zeta_{i} \partial \zeta_{j}}(\zeta)\left(z_{j}-\zeta_{j}\right)\right) Q(\zeta, z)\right\}\left(z_{i}-\zeta_{i}\right) .
\end{aligned}
$$

Therefore we have

$$
g_{i}(\zeta, \zeta)=2 \frac{\partial \rho}{\partial \zeta_{i}}(\zeta) Q(\zeta, \zeta), \quad 1 \leq i \leq n
$$

By Lemma 1, we obtain

$$
\begin{aligned}
\Omega(\zeta, z)= & \frac{1}{(2 \pi i)^{k}} \frac{\left(\sum_{j=1}^{k} 2 \frac{\partial \rho}{\partial \zeta_{j}}(\zeta) Q(\zeta, \zeta) d \zeta_{j}\right) \wedge\left(2 \sum_{j=1}^{k} \bar{\partial}_{\zeta}\left(\frac{\partial \rho}{\partial \zeta}(\zeta) Q(\zeta, \zeta)\right) \wedge d \zeta_{j}\right)^{k-1}}{F(\zeta, z)^{k} Q(\zeta, z)^{k}} \\
& +\frac{e(\zeta, z)}{F(\zeta, z)^{k} Q(\zeta, z)^{k}} \\
= & \frac{2^{k}}{(2 \pi i)^{k}} \frac{\partial \rho(\zeta) \wedge(\bar{\partial} \partial \rho)^{k-1}(\zeta)+e(\zeta, z)}{F(\zeta, z)^{k}}
\end{aligned}
$$

This completes the proof of Lemma 2. 
Now we prove the following lemma which will be used in the proof of Theorem 2 in order to calculate the principal value of the kernel function.

LEMMA 3. For $\zeta, z \in \partial V$, it holds that

$$
F(\zeta, z)-\overline{F(z, \zeta)}=O\left(|\zeta-z|^{3}\right)
$$

Proof. We may assume that $h_{1}=z_{k+1}, \ldots, h_{p}=z_{n}$. By the Taylor expansion, we have

$$
\begin{aligned}
\frac{1}{2} F(\zeta, z)= & \sum_{i=1}^{k} \frac{\partial \rho}{\partial \zeta_{i}}(\zeta)\left(z_{i}-\zeta_{i}\right) \\
& +\frac{1}{2} \sum_{i, j=1}^{k} \frac{\partial^{2} \rho}{\partial \zeta_{i} \partial \zeta_{j}}(\zeta)\left(z_{i}-\zeta_{i}\right)\left(z_{j}-\zeta_{j}\right) \\
= & \sum_{i=1}^{k}\left(\frac{\partial \rho}{\partial \zeta_{i}}(z)+\sum_{j=1}^{k} \frac{\partial^{2} \rho}{\partial \zeta_{i} \partial \zeta_{j}}(z)\left(\zeta_{j}-z_{j}\right)\right. \\
& +\frac{1}{2} \sum_{i, j=1}^{k} \frac{\partial^{2} \rho}{\partial \zeta_{i} \partial \zeta_{j}}(\zeta)\left(\zeta_{i}-z_{i}\right)\left(\zeta_{j}-z_{j}\right)+O\left(|\zeta-z|^{3}\right)
\end{aligned}
$$

On the other hand we have

$$
\begin{aligned}
\frac{1}{2} \overline{F(\zeta, z)}= & \sum_{i=1}^{k} \frac{\partial \rho}{\partial \bar{\zeta}_{i}}(\zeta)\left(\bar{\zeta}_{i}-\bar{z}_{i}\right) \\
& +\frac{1}{2} \sum_{i, j=1}^{k} \frac{\partial^{2} \rho}{\partial \bar{\zeta}_{i} \partial \bar{\zeta}_{j}}(\zeta)\left(\bar{\zeta}_{i}-\bar{z}_{i}\right)\left(\bar{\zeta}_{j}-\bar{z}_{j}\right)
\end{aligned}
$$

Thus we obtain 


$$
\begin{aligned}
\frac{1}{2}(F(\zeta, z)-\overline{F(z, \zeta))} \\
=-\sum_{i=1}^{k} \frac{\partial \rho}{\partial \zeta_{i}}(z)\left(\zeta_{i}-z_{i}\right)-\sum_{i=1}^{k} \frac{\partial \rho}{\partial \bar{\zeta}_{i}}(z)\left(\bar{\zeta}_{i}-\bar{z}_{i}\right) \\
\quad-\frac{1}{2} \sum_{i, j=1}^{k} \frac{\partial^{2} \rho}{\partial \zeta_{i} \partial \zeta_{j}}(z)\left(\zeta_{i}-z_{i}\right)\left(\zeta_{j}-z_{j}\right) \\
\quad-\frac{1}{2} \sum_{i, j=1}^{k} \frac{\partial^{2} \rho}{\partial \bar{\zeta}_{i} \partial \bar{\zeta}_{j}}(z)\left(\bar{\zeta}_{i}-\bar{z}_{i}\right)\left(\bar{\zeta}_{j}-\bar{z}_{j}\right) \\
\quad-\sum_{i, j=1}^{k} \frac{\partial^{2} \rho}{\partial \zeta_{i} \partial \bar{\zeta}_{j}}(z)\left(\zeta_{i}-z_{i}\right)\left(\bar{\zeta}_{j}-\bar{z}_{j}\right)+O\left(|\zeta-z|^{3}\right) \\
=-\rho(\zeta)+\rho(z)+O\left(|\zeta-z|^{3}\right)=O\left(|\zeta-z|^{3}\right)
\end{aligned}
$$

This completes the proof of Lemma 3.

2. The principal value of the kernel function. Let $z \in \partial V$ and $f$ be a continuous function on $\partial V$. If

$$
\lim _{\varepsilon \rightarrow 0+} \int_{\partial V \cap\{\zeta:|g(\zeta, z)|>\varepsilon\}} f(\zeta) \Omega(\zeta, z)
$$

exists, then we stand for the above limit by

$$
\text { P.V. } \int_{\partial V} f(\zeta) \Omega(\zeta, z)
$$

Now we are going to prove the following theorem which was obtained by Kerzman and Stein [9] when $k=n$.

THEOREM 2. For $z \in \partial V$, it holds that

$$
\text { P.V. } \int_{\zeta \in \partial V} \Omega(\zeta, z)=\frac{1}{2} .
$$

Proof. Let $q \in \partial V$ be fixed. We may assume that for $\delta>0$ sufficiently small,

$$
\widetilde{V} \cap S_{q, \delta}=\left\{z \in S_{q, \delta}: z_{k+1}=\cdots=z_{n}=0\right\} .
$$


For $z \in \widetilde{V} \cap S_{q, \delta}$, we have

$$
\begin{aligned}
\rho(z)=2 \operatorname{Re}\left(\sum_{i=1}^{k} \frac{\partial \rho}{\partial z_{i}}(q)\left(z_{i}-q_{i}\right)\right. & \\
& \left.+\frac{1}{2} \sum_{i, j=1}^{k} \frac{\partial^{2} \rho}{\partial z_{i} \partial z_{j}}(q)\left(z_{i}-q_{i}\right)\left(z_{j}-q_{j}\right)\right) \\
& +\sum_{i, j=1}^{k} \frac{\partial^{2} \rho}{\partial z_{i} \partial \bar{z}_{j}}(q)\left(z_{i}-q_{i}\right)\left(\bar{z}_{j}-\bar{q}_{j}\right)+O\left(|z-q|^{3}\right) .
\end{aligned}
$$

For simplicity, we may assume that $q=0$. By (1), we can find a new local coordinate system $w_{1}, \ldots, w_{n}$ by letting

$$
w_{1}=2 \sum_{i=1}^{k} \frac{\partial \rho}{\partial z_{i}}(0) z_{i}+\sum_{i, j=1}^{k} \frac{\partial^{2} \rho}{\partial z_{i} \partial z_{j}}(0) z_{i} z_{j}
$$

and choosing $w_{1}, \ldots, w_{n}$ suitably such that $w(0)=0$. Then we have

$$
\rho(w)=\operatorname{Re} w_{1}+\sum_{i, j=1}^{k} \frac{\partial^{2} \rho}{\partial w_{i} \partial \bar{w}_{j}}(0) w_{i} \bar{w}_{j}+O\left(|w|^{3}\right) .
$$

By a unitary transformation $w_{i}^{\prime}=\left(w_{1}^{\prime}, \ldots, w_{n}^{\prime}\right)$, we obtain

$$
\rho\left(w^{\prime}\right)=\operatorname{Re}\left(\sum_{j=1}^{k} a_{j} w_{j}^{\prime}\right)+\sum_{i=1}^{k} \frac{\partial^{2} \rho}{\partial w_{i}^{\prime} \partial \bar{w}_{i}^{\prime}}(0)\left|w_{i}^{\prime}\right|^{2}+O\left(\left|w^{\prime}\right|^{3}\right),
$$

where $\left(a_{1}, \ldots, a_{k}\right)$ is a non zero vector. Again we can find a new local coordinate system $\zeta=\left(\zeta_{1}, \ldots, \zeta_{n}\right)$ such that

$$
\rho(\zeta)=\operatorname{Re} \zeta_{1}+\sum_{i=1}^{k}\left|\zeta_{i}\right|^{2}+O\left(|\zeta|^{3}\right)
$$

We set $\zeta_{j}=x_{j}+i y_{j}$. Then we have

$$
\begin{aligned}
& \partial \rho(0) \wedge(\bar{\partial} \partial \rho)^{k-1}(0) \\
&=\left(\sum_{j=1}^{k} \frac{\partial \rho}{\partial \zeta_{j}}(0) d \zeta_{j}\right) \wedge\left(\sum_{i=1}^{k} \frac{\partial^{2} \rho}{\partial \zeta_{i} \partial \bar{\zeta}_{i}}(0) d \bar{\zeta}_{i} \wedge d \zeta_{i}\right)^{k-1}
\end{aligned}
$$

Since $d \rho=0$ on $\partial V$, we have

$$
\sum_{j=1}^{k} \frac{\partial \rho}{\partial x_{j}}(0) d x_{j}+\sum_{j=1}^{k} \frac{\partial \rho}{\partial y_{j}}(0) d y_{j}=0
$$


Therefore we have

$$
\begin{aligned}
\partial \rho(0) & \wedge(\bar{\partial} \partial \rho)^{k-1}(0) \\
= & \frac{i}{2} \sum_{j=1}^{k}\left(\frac{\partial \rho}{\partial x_{j}}(0) d y_{j}-\frac{\partial \rho}{\partial y_{j}}(0) d x_{j}\right) \\
& \wedge(2 i)^{k-1}\left(\sum_{j=2}^{k} \frac{\partial^{2} \rho}{\partial w_{j} \partial \bar{w}_{j}}(0) d x_{j} \wedge d y_{j}\right)^{k-1} \\
= & 2^{k-2} i^{k}(k-1) ! d y_{1} \wedge d x_{2} \wedge d y_{2} \wedge \cdots \wedge d x_{k} \wedge d y_{k} .
\end{aligned}
$$

Thus, by Lemma 2, we obtain

(4)

$$
\Omega(\zeta, z)
$$

$$
=\frac{2^{k-2}(k-1) ! d y_{1} \wedge d x_{2} \wedge d y_{2} \wedge \cdots \wedge d x_{k} \wedge d y_{k}+O(|\zeta|+|\zeta-z|)}{\pi^{k} F(\zeta, z)^{k}} .
$$

Let $\nu$ be the unit inner normal of $\partial V$ at 0 . Then we have

$$
\nu=-\left(\frac{\partial \rho}{\partial x_{1}}(0), \frac{\partial \rho}{\partial y_{1}}(0), \ldots, \frac{\partial \rho}{\partial y_{n}}(0)\right)=(-1,0, \ldots, 0) .
$$

We set, for $\delta>0$ sufficiently small, $z=\nu \delta$. Then we have

$$
\begin{aligned}
F(\zeta, z) & -F(\zeta, 0) \\
= & \sum_{i=1}^{k}\left(-\zeta_{i}+\nu_{i} \delta\right)\left(2 \frac{\partial \rho}{\partial \zeta_{i}}(\zeta)+\sum_{j=1}^{k} \frac{\partial^{2} \rho}{\partial \zeta_{i} \partial \zeta_{j}}(\zeta)\left(-\zeta_{j}+\nu_{j} \delta\right)\right) \\
& -\sum_{i=1}^{k}\left(-\zeta_{i}\right)\left(2 \frac{\partial \rho}{\partial \zeta_{i}}(\zeta)+\sum_{j=1}^{k} \frac{\partial^{2} \rho}{\partial \zeta_{i} \partial \zeta_{j}}(\zeta)\left(-\zeta_{j}\right)\right) \\
= & \sum_{i=1}^{k} 2 \nu_{i} \delta \frac{\partial \rho}{\partial \zeta_{i}}(\zeta) \\
& +\sum_{i, j=1}^{k}\left(\nu_{i} \nu_{j} \delta^{2}-\nu_{i} \zeta_{j} \delta-\nu_{j} \zeta_{i} \delta\right) \frac{\partial^{2} \rho}{\partial \zeta_{i} \partial \zeta_{j}}(\zeta) \\
= & -\delta+O\left(\delta|\zeta|+\delta^{2}\right) .
\end{aligned}
$$

On the other hand we have

$$
F(0, \zeta)=\sum_{i=1}^{k} \zeta_{i}\left(2 \frac{\partial \rho}{\partial \zeta_{i}}(0)+\sum_{j=1}^{k} \frac{\partial^{2} \rho}{\partial \zeta_{i} \partial \zeta_{j}}(0) \zeta_{j}\right)=\zeta_{1}=x_{1}+i y_{1}
$$


and

$$
0=\rho(\zeta)=x_{1}+\sum_{i=2}^{k}\left|\zeta_{i}\right|^{2}+O\left(|\zeta|^{3}\right)
$$

Thus we obtain

$$
F(0, \zeta)=-\sum_{i=1}^{k}\left|\zeta_{i}\right|^{2}+i y_{1}+O\left(|\zeta|^{3}\right)
$$

Taking account of Lemma 3, we have

$$
\begin{aligned}
F(\zeta, z) & =F(\zeta, 0)-\delta+O\left(|\zeta| \delta+\delta^{2}\right) \\
& =\overline{F(0, \zeta)}-\delta+O\left(|\zeta|^{3}+|\zeta| \delta+\delta^{2}\right) \\
& =-i y_{1}-\sum_{i=2}^{k}\left|\zeta_{i}\right|^{2}-\delta+O\left(|\zeta|^{3}+|\zeta| \delta+\delta^{2}\right)
\end{aligned}
$$

We set

$$
\beta(0, \varepsilon)=\lim _{\delta \rightarrow 0} \int_{\{\zeta \in \partial V:|F(\zeta, 0)|<\varepsilon\}} \frac{K_{V}(\zeta, \nu \delta)}{F(\zeta, \nu \delta)^{k}} .
$$

Then, by (4), we have

$$
\beta(0, \varepsilon)=\lim _{\delta \rightarrow 0} \int_{\{\zeta \in \partial V:|F(\zeta, 0)|<\varepsilon\}} \frac{2^{k-2}(k-1) ! \sigma(d \zeta)+O(|\zeta|+\delta)}{\pi^{k} F(\zeta, \nu \delta)^{k}}
$$

where $\sigma(d \zeta)$ is the surface element of $\{\zeta \in \partial V:|F(\zeta, 0)|<\varepsilon\}$. We set $\zeta^{\prime}=\left(\zeta_{2}, \ldots, \zeta_{k}\right)$. It holds from $(3)$ that $\left(\partial \rho / \partial x_{1}\right)(0) \neq 0$. Therefore by the implicit function theorem, $\rho=0$ can be represented by $x_{1}=\varphi\left(y_{1}, \zeta^{\prime}\right)$, where $\varphi$ is a smooth function satisfying $\varphi(0,0)=$ 0 . Thus we have $x_{1}=O\left(\left|y_{1}\right|+\left|\zeta^{\prime}\right|\right)$. Hence there exists a constant $c_{1}>0$ such that

$$
||\left|\zeta^{\prime}\right|^{2}+i y_{1}|-| F(\zeta, 0)|| \leq c_{1}\left(\left|\zeta^{\prime}\right|+\left|y_{1}\right|\right)^{3} .
$$

Therefore, for $\varepsilon>0$ sufficiently small, we have

$$
\begin{aligned}
& \left\{\zeta \in \partial V:\left.|| \zeta^{\prime}\right|^{2}+i y_{1} \mid<\frac{\delta}{2}\right\} \\
& \quad \subset\{\zeta \in \partial V:|F(\zeta, 0)|<\varepsilon\} \subset\left\{\zeta \in \partial V:\left.|| \zeta^{\prime}\right|^{2}+i y_{1} \mid<\frac{3}{2} \varepsilon\right\} .
\end{aligned}
$$

Thus we have

$$
\beta(0, \varepsilon)=\frac{2^{k-2}(k-1) !}{\pi^{k}} \lim _{\delta \rightarrow 0} \int_{\{\zeta \in \partial V:|F(\zeta, 0)|<\varepsilon\}} \frac{\sigma(d \zeta)}{F(\zeta, \nu \delta)^{k}},
$$


provided the limit of the right-hand side exists. We set $A=\delta+\left|\zeta^{\prime}\right|^{2}+$ $i y_{1}$. Then we have $F(\zeta, \nu \delta)=A+\mathscr{E}$ where $\mathscr{E}=O\left(|\zeta|^{3}+\delta|\zeta|+\delta^{2}\right)$. Then for some constant $c_{2}>0$, it holds that

$$
\left|\frac{\mathscr{E}}{A}\right| \leq c_{2}\left(\left|\zeta^{\prime}\right|+\left|y_{1}\right|+\delta\right) \text {. }
$$

Thus if we choose $\delta$ and $\varepsilon$ sufficiently small, then we have

$$
\left|\frac{\mathscr{E}}{A}\right| \leq \frac{1}{2}
$$

On the other hand we have

$$
\frac{1}{F(\zeta, \nu \delta)^{k}}=\frac{1}{(A+\mathscr{E})^{k}}=\frac{1}{A^{k}}+\mathscr{E}^{\prime},
$$

where $\mathscr{E}^{\prime}$ satisfies, for some constant $c_{3}>0$,

$$
\left|\mathscr{E}^{\prime}\right| \leq \frac{c_{3}\left(\left|\zeta^{\prime}\right|+\left|y_{1}\right|+\delta\right)}{|A|^{k}}
$$

It holds that

$$
\int_{\left\{\left.\left|i y_{1}+\right| \zeta^{\prime}\right|^{2}<\varepsilon\right\}} \frac{\left|\zeta^{\prime}\right|+\left|y_{1}\right|+\delta}{|A|^{k}} \sigma(d \zeta) \rightarrow 0 \quad(\text { as } \delta \rightarrow 0, \varepsilon \rightarrow 0) .
$$

Thus we obtain

$$
\beta(0, \varepsilon)=\frac{2^{k-2}(k-1) !}{\pi^{k}} \lim _{\delta \rightarrow 0} \int_{\left\{\left.\left|i y_{1}+\right| \zeta^{\prime}\right|^{2}<\varepsilon\right\}} \frac{\sigma(d \zeta)}{\left(i y_{1}+\left|\zeta^{\prime}\right|^{2}+\delta\right)^{k}} .
$$

The dilation $\left(y-1, \zeta^{\prime}\right) \rightarrow\left(\delta y_{1}, \sqrt{\delta} \zeta^{\prime}\right)$ gives

$$
\lim _{\varepsilon \rightarrow 0} \beta(0, \varepsilon)=\frac{2^{k-2}(k-1) !}{\pi^{k}} \lim _{R \rightarrow \infty} \int_{\left\{\left.\left|i y_{1}+\right| \zeta^{\prime}\right|^{2} \mid<R\right\}} \frac{\sigma(d \zeta)}{\left(i y_{1}+\left|\zeta^{\prime}\right|^{2}+1\right)^{k}} .
$$

The calculation of this limit is contained in Korànyi and Vagi [10] as follows. Consider the integral

$$
I(\varepsilon, R)=\int_{\left\{\varepsilon<\left.\left|i y_{1}+\right| \zeta^{\prime}\right|^{2} \mid<R\right\}} \frac{\sigma(d \zeta)}{\left(i y_{1}+\left|\zeta^{\prime}\right|^{2}+1\right)^{k}}, \quad 0<\varepsilon<R .
$$

Introduce polar coordinates in $C^{k-1}$,

$$
\rho=\left|\zeta^{\prime}\right|, \quad \omega=\frac{\zeta^{\prime}}{\left|\zeta^{\prime}\right|}, \quad \sigma(d \zeta)=\rho^{2 k-3} d y_{1} d \rho d \omega
$$

where $d \omega$ is the surface element in the unit sphere $S^{2 k-3}$ in $C^{k-1}$. We denote the volume of the unit sphere $S^{2 k-3}$ by $\left|S^{2 k-3}\right|$. Next we make the variable change

$$
u=\rho^{2}, \quad d u=2 \rho d \rho
$$


and then introduce polar coordinates in the $u, y_{1}$ halfplane,

$$
\begin{aligned}
& u+i y_{1}=s e^{i \theta}, \quad-\frac{\pi}{2} \leq \theta \leq \frac{\pi}{2}, \quad \varepsilon<s<R, \\
& d u d y_{1}=s d s d \theta .
\end{aligned}
$$

We find

$$
\begin{aligned}
I(\varepsilon, R) & =\left|S^{2 k-3}\right| \int_{\left\{\varepsilon^{2}<y_{1}^{2}+\rho^{4}<R^{2}\right\}} \frac{\rho^{2 k-3} d \rho d y_{1}}{\left(1+\rho^{2}+i y_{1}\right)^{k}} \\
& =\frac{\left|S^{2 k-3}\right|}{2} \int_{\left\{\varepsilon^{2}<y_{1}^{2}+u^{2}<R, 0 \leq u\right\}} \frac{u^{k-1} d u d y_{1}}{\left(1+u+i y_{1}\right)^{k}} \\
& =\frac{\left|S^{2 k-3}\right|}{2} \int_{-\pi / 2}^{\pi / 2} \cos ^{k-2} \theta d \theta \int_{\varepsilon}^{R} \frac{s^{k-1} d s}{\left(1+s e^{i \theta}\right)^{k}} .
\end{aligned}
$$

The variable change $\theta \rightarrow \theta-\frac{\pi}{2}, s \rightarrow \frac{1}{\rho}$ finally gives

$$
I(\varepsilon, R)=\frac{\left|S^{2 k-3}\right|}{2} \int_{0}^{\pi} \sin ^{k-2} \theta d \theta \int_{1 / R}^{1 / \varepsilon} \frac{d \rho}{\rho\left(\rho-i e^{i \theta}\right)} .
$$

Then Korànyi and Vagi ([10], Lemma 6.2, p. 613) gives

$$
\lim _{\substack{\varepsilon \rightarrow 0+\\ R \rightarrow \infty}} I(\varepsilon, R)=\frac{\pi^{k-1}}{(k-2) !} \cdot \frac{\pi}{2^{k-1}(k-1) !}=\frac{\pi^{k}}{2^{k-1}(k-1) !} .
$$

Hence we obtain

$$
\lim _{\varepsilon \rightarrow 0+} \beta(0, \varepsilon)=\frac{1}{2},
$$

which completes the proof of Theorem 2 .

3. The continuous extension to the boundary. The following proposition is proved essentially by Adachi [1] (cf. Henkin [7]). But for the comparison with the principal value integral, we give the sketch of the proof.

Proposition 1. Define, for $z \in \bar{D} \mid \partial V$,

$$
H(z)=\int_{\zeta \in \partial V} \Omega(\zeta, z) .
$$

Then the function

$$
\widetilde{H}(z)= \begin{cases}H(z) & (z \in \bar{D} \mid \partial V) \\ 1 & (z \in \partial V)\end{cases}
$$

belongs to $A(D)$. 
Proof. Since $H(z)$ is holomorphic in $\bar{D} \mid \partial V$, it is sufficient to show that, for $z^{0} \in \partial V$,

$$
\lim _{z \rightarrow z^{0}, z \in \bar{D} \mid \partial V} H(z)=1 .
$$

We may assume that

$$
\widetilde{V} \cap S_{z^{0}, \sigma_{1}}=\left\{z \in S_{z^{0}, \sigma_{1}}: z_{k+1}=\cdots=z_{n}=0\right\} .
$$

By (1), we may assume, without loss of generality, that $\left(\partial \rho / \partial \zeta_{1}\right)\left(z^{0}\right) \neq$ 0 . For $z \in S_{z^{0}, \sigma_{1}}$, we consider the system of equations for $\zeta^{0}=$ $\left(\zeta_{1}^{0}, \ldots, \zeta_{n}^{0}\right)$ of the following form:

$$
\left\{\begin{array}{l}
\sum_{i=1}^{n} \frac{\partial \rho}{\partial \zeta_{i}}\left(\zeta^{0}\right)\left(\zeta_{i}^{0}-z_{i}\right)=0, \\
\zeta_{i}^{0}=z_{i}(i=2, \ldots, k), \quad \zeta_{k+1}^{0}=\cdots=\zeta_{n}^{0}=0 .
\end{array}\right.
$$

We set $\varepsilon=\left(\left|z_{k+1}\right|^{2}+\cdots+\left|z_{n}\right|^{2}\right)^{1 / 2}$. Then by Adachi [1], there exist positive constants $\sigma_{2}\left(<\sigma_{1}\right), \gamma_{1}$ and $\gamma_{2}$ such that for any $\sigma \leq \sigma_{2}$ and any $z \in S_{z^{0}, \sigma / 2} \cap(\bar{D} \mid \partial V)$, there exists a unique solution $\zeta^{0}=\zeta^{0}(z)$ of the system (5) which belongs to the set $S_{z^{0}, \sigma} \cap V$ and satisfies the following.

$$
\begin{gathered}
\varepsilon \leq\left|z-\zeta^{0}\right| \leq \gamma_{1} \varepsilon \\
\left|\sum_{i=1}^{n} \frac{\partial g}{\partial z_{i}}(\zeta, z)\left(\zeta_{i}^{0}-z_{i}\right)\right| \leq \gamma_{2} \varepsilon(|\zeta-z|+\varepsilon) .
\end{gathered}
$$

From the integral formula (2) we have

$$
H\left(\zeta^{0}\right)=1 .
$$

Hence it is sufficient to show that

$$
\lim _{z \rightarrow z^{0}, z \in \bar{D} \mid \partial V}\left|H(z)-H\left(\zeta^{0}\right)\right|=0 .
$$

Let $z \in S_{z^{0}, \sigma / 2} \cap(\bar{D} \mid \partial V)$. Let $V^{\prime}$ be an open subset in $\widetilde{V}$ with smooth boundary such that $\bar{V} \subset V^{\prime} \subset \bar{V}^{\prime} \subset \widetilde{V}$. By using Stokes' formula, we have

$$
H(z)=\int_{\partial V^{\prime}} \Omega(\zeta, z)-\int_{V^{\prime}-V} \bar{\partial}_{\zeta} \Omega(\zeta, z) .
$$

Define

$$
\Psi(z)=\int_{\left(V^{\prime}-V\right) \cap S_{z^{0}, \sigma}} \bar{\partial}_{\zeta} \Omega(\zeta, z) .
$$


It is sufficient to show that

$$
\lim _{z \rightarrow z^{0}, z \in \bar{D} \mid \partial V}\left|\Psi(z)-\Psi\left(\zeta^{0}\right)\right|=0
$$

We can write $\Psi(z)$ in the following form

$$
\begin{aligned}
\Psi(z)= & \int_{\left(V^{\prime}-V\right) \cap S_{z^{0}, \sigma}} \frac{A(\zeta, z)}{g(\zeta, z)^{k}} \\
& +\int_{\left(V^{\prime}-V\right) \cap S_{z^{0}, \sigma}} \sum_{j=1}^{n} \frac{\left(\zeta_{j}-z_{j}\right) B_{j}(\zeta, z)}{g(\zeta, z)^{k+1}}
\end{aligned}
$$

where $A(\zeta, z), B_{j}(\zeta, z)$ are $(k, k)$ forms that are smooth in $(\zeta, z)$ and holomorphic in $z$. By using (6), (7), there are positive constants $\gamma_{3}$ and $\gamma_{4}$ such that

$$
\begin{aligned}
& \left|\frac{d \Psi\left(\zeta^{0}+\lambda\left(z-\zeta^{0}\right)\right)}{d \lambda}\right| \lambda \mid \\
& \quad \leq \gamma_{3} \int_{\left(V^{\prime}-V\right) \cap S_{z^{0}, \sigma}} \frac{\varepsilon d V}{|g(\zeta, z)|^{k+1}} \\
& \quad+\gamma_{4} \int_{\left(V^{\prime}-V\right) \cap S_{z^{0}, \sigma}} \frac{|\zeta-z| \varepsilon(|\zeta-z|+\varepsilon) d V}{|g(\zeta, z)|^{k+2}} .
\end{aligned}
$$

By the estimates obtained by Henkin [7], we have for some constant $\gamma_{5}>0$

$$
\left|\frac{d \Psi\left(\zeta^{0}+\lambda\left(z-\zeta^{0}\right)\right)}{d \lambda}\right|_{\lambda=1} \mid \leq \gamma_{5}(\varepsilon|\log \varepsilon|+\varepsilon) .
$$

Let

$$
z(\theta)=\zeta^{0}+\theta\left(z-\zeta^{0}\right) \text { for } \theta \in[0,1] .
$$

Then the uniqueness of the solution of the system (5) implies $\zeta^{0}(z(\theta))$ $=\zeta^{0}(z)$. Therefore from (8), we have, for some constant $\gamma_{6}>0$,

$$
\begin{aligned}
& \left|\frac{d \Psi\left(\zeta^{0}+\lambda \theta\left(z-\zeta^{0}\right)\right)}{d \lambda}\right|_{\lambda=1} \mid \\
& \quad=\left|\frac{d \Psi\left(\zeta^{0}(z(\theta))+\lambda\left(z(\theta)-\zeta^{0}(z(\theta))\right)\right)}{d \lambda}\right|_{\lambda=1} \mid \\
& \quad \leq \gamma_{6}(\theta \varepsilon|\log (\theta \varepsilon)|+\theta \varepsilon) .
\end{aligned}
$$


Thus we obtain for some constant $\gamma_{7}>0$,

$$
\begin{aligned}
\left|\Psi(z)-\Psi\left(\zeta^{0}\right)\right| & =\left|\int_{0}^{1} \frac{d}{d \theta} \Psi\left(\zeta^{0}+\theta\left(z-\zeta^{0}\right)\right) d \theta\right| \\
& =\left|\int_{0}^{1} \frac{1}{\theta}\left(\frac{d \Psi\left(\zeta^{0}+\lambda \theta\left(z-\zeta^{0}\right)\right)}{d \lambda}\right)_{\lambda=1} d \theta\right| \\
& \leq \gamma_{7} \int_{0}^{1}(\varepsilon|\log \varepsilon|+\varepsilon|\log \theta|+\varepsilon) d \theta \rightarrow 0 \quad(\varepsilon \rightarrow 0),
\end{aligned}
$$

which completes the proof of Proposition 1.

4. The extension of Lipschitz functions from the boundary. In order to prove Lemma 5, we need the following lemma which is the modified version of Lemma 3.1 of Henkin [6].

LEMMA 4. Let $t=\left(t_{1}, \ldots, t_{2 k}\right) \in R^{2 k}, \varepsilon>0,0<\delta<1, t^{\prime}=$ $\left(t_{2}, \ldots, t_{2 k}\right)$. Then we have

$$
I_{1}=\int_{\left\{\delta^{2} \leq\left|t^{\prime}\right|^{2}+\varepsilon^{2} \leq 1\right\}} \frac{d t_{2} \cdots d t_{2 k}}{\left[\left(\left|t^{\prime}\right|^{2}+\varepsilon^{2}\right)^{2}+t_{2}^{2}\right]^{k / 2}} \leq \gamma \log \frac{\gamma}{\delta}
$$

where $\gamma$ is the constant which is independent of $\varepsilon$ and $\delta$.

Proof. (a) In case $\varepsilon^{2} \leq \frac{1}{2} \delta^{2}$. Since $\delta^{2} \leq\left|t^{\prime}\right|^{2}+\varepsilon^{2}$, we have $\left|t^{\prime}\right|^{2} \geq$ $\frac{1}{2} \delta^{2}$. Therefore we have

$$
I_{1} \leq \int_{\left\{\delta^{2} / 2 \leq\left|t^{\prime}\right|^{2} \leq 1\right\}} \frac{d t_{2} \cdots d t_{2 k}}{\left(\left|t^{\prime}\right|^{2}+t_{2}\right)^{k / 2}}
$$

If $k=1$, then we have

$$
I_{1} \leq \int_{\delta / 2}^{1} \frac{d t_{2}}{t_{2}} \leq \gamma \log \frac{\gamma}{\delta}
$$

If $k \geq 2$, by using polar coordinates, we have for some $\gamma_{1}>0$, 


$$
\begin{aligned}
I_{1} & \leq \gamma_{1} \int_{\delta / 2}^{1} d r \int_{0}^{\pi} \frac{r^{2 k-2} \sin ^{2 k-3} \varphi d \theta}{\left(r^{4}+r^{2} \cos ^{2} \varphi\right)^{k / 2}} \\
& =\gamma_{1} \int_{\delta / 2}^{1} d r \int_{0}^{\pi} \frac{r^{k-2} \sin ^{2 k-3} \varphi d \varphi}{\left(r^{2}+\cos ^{2} \varphi\right)^{k / 2}} \\
& \leq \gamma_{1} \int_{\delta / 2}^{1} d r \int_{0}^{\pi} \frac{\sin ^{2 k-3} \varphi}{r^{2}+\cos ^{2} \varphi} d \varphi \\
& \leq \gamma_{1} \int_{\delta / 2}^{1} d r \int_{-1}^{1} \frac{d s}{r^{2}+s^{2}}=\gamma_{1} \int_{\gamma / 2}^{1} \frac{2}{r} \tan ^{-1}\left(\frac{1}{r}\right) d r \\
& \leq \pi \gamma_{1} \int_{\delta / 2}^{1} \frac{d r}{r} \leq \gamma \log \frac{\gamma}{\delta} .
\end{aligned}
$$

(b) In case $\varepsilon^{2} \geq \frac{1}{2} \delta^{2}$. Then we have

$$
I_{1} \leq \int_{\left\{\left|t^{\prime}\right| \leq 1\right\}} \frac{d t_{2} \cdots d t_{2 k}}{\left[\left(\left|t^{\prime}\right|^{2}+\varepsilon^{2}\right)^{2}+t_{2}^{2}\right]^{k / 2}}
$$

If $k=1$, then we have for some $\gamma_{2}>0$,

$$
I_{1} \leq \gamma_{2} \int_{0}^{1} \frac{d t_{2}}{t_{2}+\delta^{2}} \leq \gamma \log \frac{\gamma}{\delta} .
$$

If $k \geq 2$, then we obtain for some $\gamma_{3}>0$,

$$
\begin{aligned}
I_{1} & \leq \gamma_{3} \int_{0}^{1} d r \int_{0}^{\pi} \frac{r^{2 k-2} \sin ^{2 k-3} \varphi d \theta}{\left[\left(r^{2}+\delta^{2}\right)^{2}+r^{2} \cos ^{2} \varphi\right]^{k / 2}} \\
& \leq \gamma_{3} \int_{0}^{1} d r \int_{0}^{\pi} \frac{\sin ^{2 k-3} \varphi d \varphi}{\left(r+\delta^{2} / r\right)^{2}+\cos ^{2} \varphi} \\
& \leq \gamma_{3} \int_{0}^{1} d r \int_{-1}^{1} \frac{d s}{\left(r+\delta^{2} / r\right)^{2}+s^{2}} \\
& \leq \pi \gamma_{3} \int_{0}^{1} \frac{d r}{\delta^{2} / r+r} \leq \frac{\pi \gamma_{3}}{2} \int_{\delta^{2}}^{1+\delta^{2}} \frac{d \lambda}{\lambda} \leq \gamma \log \frac{\gamma}{\delta} .
\end{aligned}
$$

This completes the proof of Lemma 4.

Define, for $\delta>0$,

$$
(\partial V)_{\delta}=\{w:|w-\zeta|<\delta \text { for some } \zeta \in \partial V\}
$$

Then we have 
LEMMA 5. There exists $\delta_{0}>0$ such that for any $z \in \bar{D}$ and any $\delta$ $\left(0<\delta<\delta_{0}\right)$, it holds that

$$
I_{2}=\int_{\left(S_{z, \delta_{0}} \mid S_{z, \delta}\right) \cap \partial V} \frac{\sigma(d \zeta)}{|F(\zeta, z)|^{k}} \leq \gamma \log \frac{\gamma}{\delta} .
$$

where $\gamma$ is independent of $z$ and $\delta$.

Proof. There exist positive constants $\delta_{0}$ and $\gamma_{1}$ such that

$$
-\operatorname{Re} F(\zeta, z) \geq \rho(\zeta)-\rho(z)+\gamma_{1}|\zeta-z|^{2} \text { for } z \in(\partial V)_{\delta_{0}}, \zeta \in S_{z, \delta_{0}}
$$

We may assume that $z \in(\partial V)_{\delta_{0}} \cap \bar{D}, d \rho \neq 0$ on $(\partial V)_{\delta_{0}}$, and that

$$
\widetilde{V} \cap S_{z, \delta_{0}}=\left\{w \in S_{z, \delta_{0}}: w_{k+1}=\cdots=w_{n}=0\right\} .
$$

We can find a new local coordinate system $t=\left(t_{1}, \ldots, t_{2 n}\right)$ by letting

$$
\begin{gathered}
t_{1}+i t_{2}=\rho(\zeta)-\rho(z)+i \operatorname{Im} F(\zeta, z), \\
t_{2 j-1}+i t_{2 j}=\zeta_{j}-z_{j} \text { for } j=k+1, \ldots, n,
\end{gathered}
$$

and choosing $t_{3}, \ldots, t_{2 k}$ suitably such that $t(z)=0$. Then there exist positive constants $\gamma_{2}, \gamma_{3}$ and $\gamma_{4}$ such that

$$
\begin{aligned}
& \gamma_{2}|\zeta-z|^{2} \leq|t|^{2} \leq \gamma_{3}|\zeta-z|^{2} \\
& |F(\zeta, z)| \geq \gamma_{4}\left[\left(t_{1}+|t|^{2}\right)^{2}+t_{2}^{2}\right]^{1 / 2} .
\end{aligned}
$$

Define $\varepsilon^{2}=|\rho(z)|+\left|z_{k+1}\right|^{2}+\cdots+\left|z_{n}\right|^{2}$. Taking account of the relation

$$
\begin{aligned}
\left(S_{z, \delta_{0}} \mid S_{z, \delta}\right) \cap \partial V \subset\left\{t: t_{1}=-\rho(z), \quad \gamma_{2} \delta^{2} \leq|t|^{2} \leq \gamma_{3}^{2} \delta_{0}^{2},\right. \\
\left.t_{2 j-1}+i t_{2 j}=-z_{j}(j=k+1, \ldots, n)\right\},
\end{aligned}
$$

we have, together with Lemma 4 , for some $\gamma_{5}>0$,

$$
I_{2} \leq \gamma_{5} \int_{\left\{\gamma_{2} \delta^{2} \leq|t|^{2} \leq \gamma_{3}^{2} \delta_{0}^{2}\right\}} \frac{d t_{2} \cdots d t_{2 k}}{\left[\left(t_{2}^{2}+\cdots+t_{2 k}^{2}+\varepsilon^{2}\right)^{2}+t_{2}^{2}\right]^{k / 2}} \leq \gamma \log \frac{\gamma}{\delta},
$$

which completes the proof of Lemma 5.

We set

$$
K_{V}(\zeta, z)=N_{V}(\zeta, z) \sigma(d \zeta)
$$

Then we have the following. 
LEMMA 6. Let $0<\alpha \leq 1$. Then there exists a positive constant $\delta_{0}$ such that for any $z \in \bar{D}$ and any $\delta\left(0<\delta<\delta_{0}\right)$,

$$
I_{3}=\int_{S_{z, \delta} \cap \partial V} \frac{|\zeta-z|^{\alpha}\left|N_{V}(\zeta, z)\right|}{|g(\zeta, z)|^{k}} \sigma(d \zeta) \leq \gamma \delta^{\alpha} \log \frac{\gamma}{\delta},
$$

where $\gamma$ is independent of $\delta$ and $z$.

Proof. There exists a positive constant $\gamma_{1}$ such that

$$
I_{3} \leq \gamma_{1} \int_{S_{z, \delta} \cap \partial V} \frac{|\zeta-z|^{\alpha} \sigma(d \zeta)}{|F(\zeta, z)|^{k}}
$$

Thus, by Lemma 5, we have

$$
\begin{aligned}
I_{3} & \leq \gamma_{1} \sum_{i=0}^{\infty} \int_{\left(S_{z, \delta 2^{-i}} \mid S_{z, \delta 2^{-(i+1) ~}}\right) \cap \partial V} \frac{|\zeta-z|^{\alpha} \sigma(d \zeta)}{|F(\zeta, z)|^{k}} \\
& =\sum_{i=0}^{\infty}\left(\frac{i+1}{2^{i \alpha}} \log 2\right) \gamma_{1} \delta^{\alpha}+\gamma_{1} \delta^{\alpha} \log \left(\frac{\gamma_{2}}{\delta}\right)\left(\sum_{i=0}^{\infty} \frac{1}{2^{i \alpha}}\right) \\
& \leq \gamma \delta^{\alpha} \log \frac{\gamma}{\delta},
\end{aligned}
$$

which completes the proof of Lemma 6.

Let $F$ be a closed subset of $C^{n}$. According to the definition of Stein [13], we define the Lipschitz space for $0<\alpha \leq 1$ such that

$\operatorname{Lip}(\alpha, F)=\left\{f:|f(x)| \leq M,|f(x)-f(y)| \leq M|x-y|^{\alpha}, x, y \in F\right\}$.

From the extension theorem (Stein [13], Theorem 3, p. 174), $f \in$ $\operatorname{Lip}(\alpha, F)$ can be regarded as an element of $\operatorname{Lip}\left(\alpha, C^{n}\right)$. We shall prove the following theorem which was proved by Martinelli in the case when the kernel is Bochner-Martinelli kernel (cf. Martinelli [11], Dolbeault [4]).

THEOREM 3. Let $f \in \operatorname{Lip}(\alpha, \partial V)$. Then it holds that for any $z \in$ $\partial V$,

$$
\begin{gathered}
\lim _{t \rightarrow z, t \in \bar{D} \mid \partial V} \int_{\zeta \in \partial V}(f(\zeta)-f(z)) \Omega(\zeta, t) \\
=\int_{\zeta \in \partial V}(f(\zeta)-f(z)) \Omega(\zeta, z) .
\end{gathered}
$$

Proof. Since $f \in \operatorname{Lip}(\alpha, \partial V)$, the integral of the right-hand side converges. In view of Lemma 6 , for $\varepsilon>0$, there exists $\delta>0$ such 
that for any $w \in \bar{D}$, we have

$$
\int_{S_{w, 3 \delta} \cap \partial V} \frac{|f(\zeta)-f(w)|\left|N_{V}(\zeta, w)\right|}{|g(\zeta, z)|^{k}} \sigma(d \zeta)<\varepsilon .
$$

We set

$$
\begin{gathered}
T(\zeta, w)=\frac{(f(\zeta)-f(w)) N_{V}(\zeta, w)}{g(\zeta, w)^{k}} \\
A_{\delta}=\{(\zeta, w) \in \partial V \times \bar{D}:|w-\zeta| \geq \delta\}
\end{gathered}
$$

Since $T(\zeta, w)$ is continuous on $A_{\delta}$, there exists $\rho(0<\rho<\delta)$ such that

$$
\begin{aligned}
& |T(\zeta, z)-T(\zeta, w)|<\varepsilon, \quad|f(z)-f(w)|<\varepsilon \\
& \quad \text { for }|w-z|<\rho, \quad|\zeta-z| \geq 2 \rho, \quad \zeta \in \partial V .
\end{aligned}
$$

By Proposition 1, there exists a constant $\gamma_{1}$ such that

$$
|H(t)| \leq \gamma_{1} \text { for } t \in \bar{D} \mid \partial V .
$$

Thus we have for $|t-z|<\rho, t \in \bar{D} \mid \partial V$,

$$
\begin{aligned}
& \left|\int_{\zeta \in \partial V}(f(\zeta)-f(z)) \Omega(\zeta, t)-\int_{\zeta \in \partial V}(f(\zeta)-f(z)) \Omega(\zeta, z)\right| \\
& =\left|\int_{\partial V} T(\zeta, t) \sigma(d \zeta)+(f(t)-f(z)) H(t)-\int_{\partial V} T(\zeta, z) \sigma(d \zeta)\right| \\
& \leq \gamma_{1}|f(t)-f(z)|+\int_{\partial V \mid S_{z, 2 \delta}}|T(\zeta, t)-T(\zeta, z)| \sigma(d \zeta) \\
& \quad+\int_{S_{z, 2 \delta} \cap \partial V}|T(\zeta, z)| \sigma(d \zeta)+\int_{S_{t, 3 \delta} \cap \partial V}|T(\zeta, t)| \sigma(d \zeta) \\
& \quad \leq \gamma_{1} \varepsilon+\varepsilon \int_{\partial V \mid S_{z, 2 \delta}} \sigma(d \zeta)+\varepsilon+\varepsilon \leq \gamma_{2} \varepsilon .
\end{aligned}
$$

This completes the proof of Theorem 3.

Now we shall prove the following theorem which shows that any Lipschitz function on $\partial V$ is the continuous boundary value of a function of $A(D)$ by adding $\int_{\partial V}(f(\zeta)-f(\cdot)) K_{V}(\zeta, \cdot)$.

Theorem 4. Let $f \in \operatorname{Lip}(\alpha, \partial V), 0<\alpha \leq 1$. Define

$$
\tilde{f}(z)=f(z)+\int_{\zeta \in \partial V}(f(\zeta)-f(z)) \Omega(\zeta, z) \quad \text { for } z \in \partial V,
$$


and

$$
F(z)=\int_{\zeta \in \partial V} f(\zeta) \Omega(\zeta, z) \text { for } z \in \bar{D} \mid \partial V
$$

Then the holomorphic function $F(z)$ can be extended continuously to $\bar{D}$ and it has the boundary value $\left.F\right|_{\partial V}=\tilde{f}$.

Proof. For $z \in \partial V$, we have from Theorem 3,

$$
\begin{aligned}
& \lim _{t \rightarrow z, t \in \bar{D} \mid \partial V}(F(t)-\tilde{f}(z)) \\
&=\lim _{t \rightarrow z, t \in \bar{D} \mid \partial V}\left(\int_{\partial V}(f(\zeta)-f(z)) \Omega(\zeta, t)\right. \\
&\left.\quad-\int_{\partial V}(f(\zeta)-f(z)) \Omega(\zeta, z)\right)=0 .
\end{aligned}
$$

Since $F(z)$ is holomorphic in $\bar{D} \mid \partial V, F \in A(D)$. This completes the proof of Theorem 4.

The boundary value $\tilde{f}$ is also represented by

$$
\tilde{f}(z)=\frac{1}{2} f(z)+\mathrm{P} . \mathrm{V} \cdot \int_{\partial V} f(\zeta) \Omega(\zeta, z)
$$

in view of the following.

LEMMA 7. Let $f \in \operatorname{Lip}(\alpha, \partial V)$ and $z \in \partial V$. Then we have

$$
\text { P.V. } \int_{\partial V} f(\zeta) \Omega(\zeta, z)=\int_{\partial V}(f(\zeta)-f(z)) \Omega(\zeta, z)+\frac{1}{2} f(z)
$$

Proof. We set, for $\varepsilon>0, M(\varepsilon)=\partial V \cap\{\zeta:|g(\zeta, z)|>\delta\}$. In view of Lemma 6 and Theorem 2, we obtain

$$
\begin{aligned}
\lim _{\varepsilon \rightarrow 0} \int_{M(\varepsilon)} f(\zeta) \Omega(\zeta, z)= & \lim _{\varepsilon \rightarrow 0} \int_{M(\varepsilon)}(f(\zeta)-f(z)) \Omega(\zeta, z) \\
& +f(z) \lim _{\varepsilon \rightarrow 0} \int_{M(\varepsilon)} \Omega(\zeta, z) \\
= & \int_{\partial V}(f(\zeta)-f(z)) \Omega(\zeta, z)+\frac{1}{2} f(z)
\end{aligned}
$$

This completes the proof of Lemma 7.

Now we are going to prove the following which is a main theorem in this paper. 
THEOREM 5. Let $f \in \operatorname{Lip}(\alpha, \partial V), 0<\alpha \leq 1$. If $f$ satisfies for any $z \in \partial V$,

$$
\text { P.V. } \int_{\partial V} f(\zeta) \Omega(\zeta, z)=\frac{1}{2} f(z),
$$

then there exists a function $F \in A(D)$ such that $\left.F\right|_{\partial V}=f$.

Proof. We set

$$
F(z)=\int_{\partial V} f(\zeta) \Omega(\zeta, z) \text { for } z \in \bar{D} \mid \partial V .
$$

Then $F$ can be extended continuously to $\bar{D}$ and satisfies, for $z \in \partial V$,

$$
\begin{aligned}
F(z) & =f(z)+\int_{\partial V}(f(\zeta)-f(z)) \Omega(\zeta, z) \\
& =\frac{1}{2} f(z)+\mathrm{P} . \mathrm{V} \cdot \int_{\partial V} f(\zeta) \Omega(\zeta, z)=f(z),
\end{aligned}
$$

which completes the proof of Theorem 5 .

\section{REFERENCES}

[1] K. Adachi, Continuation of bounded holomorphic functions from certain subvarieties to weakly pseudoconvex domains, Pacific J. Math., 130 (1987), 1-8.

[2] W. Alt, Singuläre Integrale mit gemischten Homogenitäten auf Mannigfaltigkeiten und Anwendungen in der Funktionentheorie, Math. Z., 137 (1974), 227256.

[3] M. Anderson and B. Berndtsson, Henkin-Ramirez formulas with weight factors, Ann. Inst. Fourier, 32 (1982), 91-110.

[4] P. Dolbeault, Théorème de Plemelj en plusieurs variables, Riv. Mat. Univ. Parma (4), 10 (1984), 47-54.

[5] T. E. Hatziafratis, Integral representation formulas on analytic varieties, Pacific J. Math., 123 (1986), 71-91.

[6] G. M. Henkin, Integral representations of functions holomorphic in strictly pseudo-convex domains and some applications, Math. USSR Sbornik, 7 (1969), $597-616$.

[7] Continuation of bounded holomorphic functions from submanifolds in general position to strictly pseudoconvex domains, Math. USSR Izvestija, 6 (1972), 536-563.

[8] G. M. Henkin and J. Leiterer, Theory of Functions on Complex Manifolds, Birkhäuser, 1984.

[9] N. Kerzman and E. M. Stein, The Szegö kernel in terms of Cauchy-Fantappie kernels, Duke Math. J., 45 (1978), 197-224.

[10] A. Korànyi and I. Vagi, Singular integrals on homogeneous spaces and some problems of classical analysis, Ann. Scuola Norm. Sup. Pisa, Sci. fis. mat. III, Ser., 25 (1971), 575-648. 
[11] E. Martinelli, Sulla determinazione di una funzione analitica di più variabili complesse in un campo, assegnatane la traccia sulla frontiera, Ann. di Mat. Pura ed Appl., 55 (1961), 191-202.

[12] E. Ramírez de Arellano, Ein Divisions problem und Randintegraldarstellungen in der Komplexen Analysis, Math. Ann., 184 (1970), 172-185.

[13] E. M. Stein, Singular integrals and differentiability properties of functions, Princeton University Press, 1970.

[14] E. L. Stout, An integral formula for holomorphic functions on strictly pseudoconvex hypersurfaces, Duke Math. J., 42 (1975), 347-356.

Received May 31, 1991.

NAGASAKI UNIVERSITY

NAGASAKI 852, JAPAN 


\title{
PACIFIC JOURNAL OF MATHEMATICS \\ Founded by \\ E. F. BECKENBACH (1906-1982) F. WolF (1904-1989)
}

\section{EDITORS}

\section{S. VARADARAJAN \\ (Managing Editor) \\ University of California \\ Los Angeles, CA 90024-1555 \\ vsv@math.ucla.edu}

\section{F. Michael Christ}

University of California

Los Angeles, CA 90024-1555

christ@math.ucla.edu

\section{Herbert Clemens}

University of Utah

Salt Lake City, UT 84112

clemens@math.utah.edu

\author{
THOMAs ENRIGHT \\ University of California, San Diego \\ La Jolla, CA 92093 \\ tenright@ucsd.edu \\ Nicholas ERcolani \\ University of Arizona \\ Tucson, AZ 85721 \\ ercolani@math.arizona.edu \\ R. FINN \\ Stanford University \\ Stanford, CA 94305 \\ finn@gauss.stanford.edu \\ VAUghan F. R. JoNeS \\ University of California \\ Berkeley, CA 94720 \\ vfr@math.berkeley.edu
}

\section{SUPPORTING INSTITUTIONS}

UNIVERSITY OF ARIZONA

UNIVERSITY OF BRITISH COLUMBIA

CALIFORNIA INSTITUTE OF TECHNOLOGY

UNIVERSITY OF CALIFORNIA

UNIVERSITY OF MONTANA

UNIVERSITY OF NEVADA, RENO

NEW MEXICO STATE UNIVERSITY OREGON STATE UNIVERSITY

\author{
UNIVERSITY OF OREGON \\ UNIVERSITY OF SOUTHERN CALIFORNIA \\ STANFORD UNIVERSITY \\ UNIVERSITY OF HAWAII \\ UNIVERSITY OF UTAH \\ WASHINGTON STATE UNIVERSITY \\ UNIVERSITY OF WASHINGTON
}

The Supporting Institutions listed above contribute to the cost of publication of this Journal, but they are not owners or publishers and have no responsibility for its content or policies.

Mathematical papers intended for publication in the Pacific Journal of Mathematics should be in typed form or offset-reproduced (not dittoed), double spaced with large margins. Please do not use built up fractions in the text of the manuscript. However, you may use them in the displayed equations. Underline Greek letters in red, German in green, and script in blue. The first paragraph must be capable of being used separately as a synopsis of the entire paper. In particular it should contain no bibliographic references. Please propose a heading for the odd numbered pages of less than 35 characters. Manuscripts, in triplicate, may be sent to any one of the editors. Please classify according to the 1991 Mathematics Subject Classification scheme which can be found in the December index volumes of Mathematical Reviews. Supply name and address of author to whom proofs should be sent. All other communications should be addressed to the managing editor, or Julie Speckart, University of California, Los Angeles, California 90024-1555.

There are page-charges associated with articles appearing in the Pacific Journal of Mathematics. These charges are expected to be paid by the author's University, Government Agency or Company. If the author or authors do not have access to such Institutional support these charges are waived. Single authors will receive 75 free reprints; joint authors will receive a total of 100 free reprints. Additional copies may be obtained at cost in multiples of 50 .

The Pacific Journal of Mathematics (ISSN 0030-8730) is published monthly except for July and August. Regular subscription rate: $\$ 200.00$ a year (10 issues). Special rate: $\$ 100.00$ a year to individual members of supporting institutions.

Subscriptions, orders for numbers issued in the last three calendar years, and changes of address should be sent to Pacific Journal of Mathematics, P.O. Box 969, Carmel Valley, CA 93924, U.S.A. Old back numbers obtainable from Kraus Periodicals Co., Route 100, Millwood, NY 10546.

The Pacific Journal of Mathematics at P.O. Box 969, Carmel Valley, CA 93924 (ISSN 0030-8730) is published monthly except for July and August. Second-class postage paid at Carmel Valley, California 93924, and additional mailing offices. Postmaster: send address changes to Pacific Journal of Mathematics, P.O. Box 969, Carmel Valley, CA 93924.

PUBLISHED BY PACIFIC JOURNAL OF MATHEMATICS, A NON-PROFIT CORPORATION

This publication was typeset using $\mathcal{A} \mathcal{S} S-\mathrm{T}_{\mathrm{E}} \mathrm{X}$, the American Mathematical Society's $\mathrm{T}_{\mathrm{E}} \mathrm{X}$ macro system.

Copyright (c) 1993 by Pacific Journal of Mathematics 


\section{PACIFIC JOURNAL OF MATHEMATICS}

Volume $158 \quad$ No. $2 \quad$ April 1993

On the extension of Lipschitz functions from boundaries of subvarieties to

201 strongly pseudoconvex domains

K. ADACHI and HiRoshi KAJIMOTO

On a nonlinear equation related to the geometry of the diffeomorphism group 223

DaVid DaI-WAi BaO, JACQUES LAFONTAINE and TUdOR S. RATIU

Fixed points of boundary-preserving maps of surfaces

ROBERT F. BROWN and BRIAN SANDERSON

On orthomorphisms between von Neumann preduals and a problem of Araki 265

L. J. BUNCE and JOHN DAVID MAITLAND WRIGHT

Primitive subalgebras of complex Lie algebras. I. Primitive subalgebras of 273 the classical complex Lie algebras

\section{V. CHEKALOV}

$L^{n}$ solutions of the stationary and nonstationary Navier-Stokes equations in 293 $R^{n}$

\section{ZHI MiN CHEN}

Some applications of Bell's theorem to weakly pseudoconvex domains

XiAO JUN HUANG

On isotropic submanifolds and evolution of quasicaustics

STANISŁAW JANECZKO

Currents, metrics and Moishezon manifolds

SHANYU JI

Stationary surfaces in Minkowski spaces. I. A representation formula

JIANGFAN LI

The dual pair $(U(1), U(1))$ over a $p$-adic field

Courtney Hughes Moen

Any knot complement covers at most one knot complement 